\title{
Alianzas y resistencias feministas en Facebook para la convocatoria del 8M en España
}

\author{
Feminist Alliances and Resistances on Facebook for \\ the $8 \mathrm{M}$ call in Spain
}

\author{
Diana Fernández-Romero (D http://orcid.org/0000-0003-2692-4474 \\ Universidad Rey Juan Carlos, España, diana.fernandez.romero@urjc.es
}

José Manuel Sánchez-Duarte (D) http://orcid.org/0000-0002-5627-2713

Universidad ReyJuan Carlos, España, josemanuel.sanchez@urjc.es

Recepción:

23/01/19

Aprobación: 07/05/19

\begin{abstract}
This article explores the political militancy exercised through a social media site by the feminist movement in Spain, analyzing the resources used to communicate and disseminate the strike call for March $8^{\text {th }}, 2018$ through the social network Facebook. For this, the level of activity of two accounts has been studied in a comparative way: one of national scope, Huelga Feminista $8 M$, and another one of regional coverage, Feminismos Madrid. The exploration was carried out on the contents published during the two weeks prior to March $8^{\text {th }}$, also including this date. We wanted to detect, by means of a quantitative study, the main frames used in Facebook messages, their contribution to knowledge on the call for a strike or on the feminist movement and the origin of discourses. We conclude that the fabric of alliances before and during the call contributed to the creation of a feminist community willing to exercise online and offline resistance and to politicize the reasons that led to the strike.
\end{abstract}

Key words: Feminist strike, March 8, frames, social networks, social movements.

Resumen: Este artículo indaga en la militancia política ejercida a través de la Red por el movimiento feminista en España, analizando los recursos empleados para difundir la convocatoria de huelga del 8 de marzo de 2018 en Facebook. Para ello, se ha estudiado la actividad de dos cuentas de forma comparativa, una de ámbito nacional, Huelga Feminista 8M, y otra de cobertura regional, Feminismos Madrid. Se exploraron los contenidos publicados durante las dos semanas previas al 8 de marzo incluyendo esta fecha. El objetivo fue detectar, mediante un estudio cuantitativo, los principales marcos de referencia (frames) utilizados en los mensajes de Facebook, su contribución al conocimiento sobre la convocatoria de paro o sobre el movimiento feminista y el origen de los discursos. Concluimos que el tejido de alianzas antes y durante la convocatoria contribuyó a la creación de una comunidad feminista dispuesta a ejercer resistencia online y offline, y a politizar los motivos que llevaron a la huelga.

Palabras clave: Huelga feminista, 8 de marzo, frames, redes sociales, movimientos sociales. 


\section{Introducción}

Los principales avances en el ámbito del activismo feminista en los últimos años provienen de la potencia de las alianzas y de los diálogos feministas en la Red (Zafra, 2018: 12). En el ciberespacio se promueven nuevas prácticas, identidades y posibilidades de contacto (Sánchez-Duarte y FernándezRomero, 2017). Internet es un espacio fundamental para desarrollar, difundir y vincularse con ideas feministas, para establecer contactos y aumentar la concienciación, además de producir nuevas identidades y prácticas (Favaro, 2018: 158). Un termómetro de ello lo constituirían la diversidad y la creatividad que, según Dean y Aune (2015: 391), siguen caracterizando el activismo feminista en la Europa del siglo XXI.

Pero también Internet es un contexto donde se ha desarrollado una fuerte misoginia en red o interconectada (Banet-Weiser y Miltner, 2016), producto de las estructuras, los códigos y los discursos patriarcales que aún se sustentan y transitan por las redes. El odio virtual convive con la persistencia de imágenes pornográficas, violentas y humillantes de las mujeres, que, como alerta Braidotti (2004: 123), todavía circulan “a través de estos productos tecnológicos supuestamente "innovadores".

Asimismo, en el ciberespacio los relatos posmachistas sobre violencia contra las mujeres han cooptado el lugar de la víctima de violencia de género. Ello se realiza por medio de estrategias discursivas de la política de la posverdad: en vez de argumentar con hechos o datos objetivos la supuesta discriminación de los hombres por la llamada "ideología de género", se recurre a la capitalización de las emociones para tratar de demostrar una situación opresiva para ellos y negar así las causas estructurales de la violencia (Núñez-Puente y Gámez-Fuentes, 2017).

Los nuevos medios, señala Wilding (2004: 145), existen dentro de un marco social insertado en ambientes económicos, políticos y culturales "que son profundamente sexistas y racistas". La Red no borra las jerarquías (Sánchez-Duarte, 2016). La atención al género en el análisis de los movimientos sociales revela cómo las interacciones mediadas digitalmente son en extremo hostiles para las mujeres, disminuyendo aún más su participación y sus posibilidades de liderazgo (Flesher y Gillan, 2017: 396).

Por ello, "es un acto radical insertar la palabra feminismo en el ciberespacio e intentar interrumpir el flujo de los códigos masculinos al declarar abiertamente la intención de mestizar, hibridar, provocar y dislocar el orden masculino de las cosas en el entorno de la Red" (Wilding, 2004: 146). La desarticulación de ese orden pasa por una lucha colectiva feminista en 
lugar del empoderamiento y la resiliencia individualista que promueve el posfeminismo.

Como indica McRobbie (2017: 323), en la cultura popular hay una reacción frente al feminismo que se muestra como algo envejecido y superfluo, mientras que se potencian las ideas de libertad y libre elección unidas a la categoría de "mujer joven". Este es un discurso que se apropia de la cultura y los medios digitales primando lo estético, lo momentáneo e incluso lo más personal (Reverter, 2013: 457). Favaro (2017) detecta una web muy comercializada y profundamente cimentada en posicionar a las mujeres como sujetos y, a la vez, como objetos de consumo.

En este contexto, el ciberfeminismo debe ser, sobre todo, reactivo y relacional (Pérez y Revuelta-Domínguez, 2008: 285). Los recursos de la Red han de aprovecharse para articular estrategias colaborativas que conecten proyectos de mujeres en distintas partes del mundo, con el fin de promover estrategias de lucha conjuntas para superar las diversas exclusiones y la desigualdad (Perdomo, 2016: 182).

En palabras de Arendt (en Gerbaudo, 2012), la acción política nunca ocurre aisladamente, sino que siempre requiere de la construcción de un sentido de "unidad" entre los involucrados, que se manifiesta en lo que llama un "espacio de apariencia", el cual surge dondequiera que las personas estén juntas en la forma de hablar y actuar.

El componente afectivo que atraviesa los discursos masivos que circulan por la Red resulta fundamental para generar una hermandad, una unión o una comunidad a partir de los afectos y las adhesiones que promueven esos discursos (Keller, 2015; Núñez-Puente y Fernández-Romero, 2017). Precisamente la sororidad y lo comunal fueron pilares esenciales de las producciones activistas digitales en torno al paro feminista del 8 de marzo de 2017, en las que el discurso testimonial sobre la violencia supo rentabilizar esa vinculación afectiva y llamar a una convocatoria para las mujeres desde la mujeres (Fernández-Romero y Núñez-Puente, 2017).

Evans y Chamberlain (2015) destacan la importancia de las redes sociales para crear y mantener una comunidad feminista. Sin embargo, al tiempo que reconocen el valor de dichas redes para acercar a las personas al feminismo y la importancia de la resistencia feminista al racismo y el sexismo en línea, también plantean preguntas sobre las formas en que las redes sociales pueden perpetuar las jerarquías entre las feministas.

En otro trabajo (Sánchez-Duarte y Fernández-Romero, 2017) hemos visto cómo las redes sociales han facilitado la movilización colectiva feminista y la creación de fuertes coaliciones para llevar a cabo acciones de presión 
muy efectivas, gracias a su difusión. Las activistas valoran ese potencial para generar comunidad y democratizar la divulgación y la visibilidad de sujetos feministas más diversos. Pero, a su vez, son autocríticas con sus prácticas, planteando la necesidad de reapropiarse del discurso feminista en redes sociales y concebir a Internet como un espacio en disputa en donde activar modos de compromiso emprendedores.

Esto supondría superar la "interpasividad" o la evitación de una acción real para ejercer una resistencia activa (Zizek, 1997: 21), una inercia que es promovida por la proliferación, distribución, aceleración e intensificación de la oportunidad y del acceso comunicativo por medio de la tecnología (Dean, 2005: 53). Sortear esta inmovilidad impulsaría la politización de asuntos para contribuir a mejorar la gobernabilidad democrática o la resistencia.

En este artículo interesa analizar cuáles fueron las estrategias del activismo feminista en Red para lograr adhesiones a la huelga del 8 de marzo de 2018 en España, en una cita que reunía características especiales por ser el primer paro feminista de 24 horas. Nuestro objetivo fue estudiar los diálogos y las alianzas que establecieron las feministas organizadas a través de dos cuentas en Facebook: una de ámbito nacional, como Huelga Feminista $8 \mathrm{M}$, y otra de ámbito regional, como Feminismos Madrid.

En ambas cuentas exploramos en qué encuadres o frames se inscribían mayoritariamente los post. Por otro lado, nos planteamos qué aportación al conocimiento sobre la huelga o sobre el movimiento feminista podría estar implicando ese contenido. Por último, nuestra intención fue desentrañar el origen de las publicaciones, con el fin de comprender si la información fue de creación propia o ajena.

Pretendemos entender si el contacto digital dio lugar a una "proximidad virtual” (Gerbaudo, 2012: 32) entre quienes apoyaron la huelga, para promover así el desarrollo de identidades colectivas, capaces de politizar la convocatoria y de activar la resistencia al patriarcado.

\section{Objetivos e hipótesis}

La convocatoria de huelga feminista -laboral, estudiantil, de cuidados y de consumo- el 8 de marzo de 2018 (8M) supuso un reto para las activistas en España, donde, de forma inédita, el paro se convocó oficialmente por algunos sindicatos minoritarios durante 24 horas. Las acciones precedentes en el contexto español de la "Plataforma $7 \mathrm{~N}$, Contra las Violencias Machistas” (Núñez-Puente y Fernández-Romero, 2017) y el éxito de respaldo del 8 de marzo de 2017, impulsado por movimientos internacionales 
como "Women's March" o el de Argentina "Ni Una Menos", asentaban un contexto favorecedor para la lucha feminista. Sin embargo, la utilización estratégica de las alianzas y los diálogos presenciales, pero en especial digitales, se convertía en una piedra angular para fomentar el amplio seguimiento de la huelga.

Como objetivo principal queremos explorar cómo el feminismo se movilizó en España para promover un paro sin precedentes a través de la red social Facebook en la cuenta Huelga Feminista $8 \mathrm{M}$ de ámbito nacional (https://www.facebook.com/HuelgaFeminista/) ${ }^{1}$ y en la de Feminismos Madrid, que se ciñe a una región concreta (https://www.facebook.com/ FeminismosMad/). ${ }^{2}$ Se eligió esta red social porque es la más utilizada en el mundo (vincos.it, 2018) y en España (CIS, 2018; IAB Spain, 2018).

En los días previos al análisis se hizo un rastreo en Facebook, Twitter e Instagram, con base en el cual concluimos que Facebook era el espacio donde se estaba promoviendo una mayor conversación para difundir la huelga. No obstante, partimos de la premisa (y con cierta cautela) de que las redes sociales de propiedad corporativa pueden implicar riesgos por el control de los datos, la opacidad o la observación o la censura de contenidos para el ciberactivismo y los movimientos sociales en línea (Flesher y Gillan, 2017: 389).

Como objetivos específicos nos planteamos hacer un seguimiento de estas dos cuentas en Facebook, entre el 22 de febrero y el 8 de marzo de 2018, para explorar las publicaciones a la luz de tres variables. La idea era conocer, por un lado, el encuadre o frame mayoritario de los post, esto es: si el marco predominante era el logístico-movilizador, el emocional, el didáctico o el noticioso. Por otro lado, se pretendía estudiar la contribución de estos contenidos al conocimiento sobre la convocatoria de paro o sobre el movimiento feminista. Finalmente, nos interesaba analizar qué fuentes eran las más recurrentes de dichos contenidos: si la creación era propia, si se acudió a los medios de comunicación o si la procedencia partió de entidades o asociaciones feministas.

Como primera hipótesis, consideramos que los mensajes que circularon por la Red previos y durante el $8 \mathrm{M}$ en ambas cuentas podrían haber adquirido una alta densidad. Esta premisa confrontaría, en este caso concreto, con la idea de Dean (2005: 52-53) del fetichismo tecnológico y la fantasía de participación en el flujo comunicativo de la Red. Dean (2005) señala que dicha fantasía es impulsada por la profusión de mensajes que

1 Esta cuenta registraba 13.503 seguidores en junio de 2018.

2 Feminismos Madrid tenía el respaldo de 3.846 seguidores en junio de 2018. 
se diluyen en Internet por su escaso o nulo contenido político. Por eso la participación digital no debe tomarse como indicador de la fuerza de un movimiento, y los datos han de delimitarse con cuidado (Flesher y Gillan, 2017: 395). La puesta en circulación de mensajes con intención activista no implicaría necesariamente, según Dean (2005), activismo político. Para que esto fuera así, sería preciso que esos mensajes elaboraran propuestas con posibilidad de politizarse, evitando la formación pospolítica del "capitalismo comunicativo".

Partimos también de la hipótesis de que los mensajes difundidos por Huelga Feminista 8M y Feminismos Madrid contribuyeron a la politización de las reivindicaciones que promovieron la huelga. No obstante, pensamos que por sus características, la cuenta Huelga Feminista 8M inscribió sus mensajes mayormente en un encuadre movilizador y, por tanto, su información suscitó una menor aportación al conocimiento: los discursos fueron sobre todo promotores de la difusión de convocatorias en el territorio nacional, sin poder profundizar en el argumentario de la huelga.

Sostenemos que la conversación pudo estar más presente en la cuenta de Feminismos Madrid, considerando la menor urgencia para difundir convocatorias en todo el país y la posibilidad de concentrar más esfuerzos en explicar los motivos de la huelga. Esto supondría una diferente apelación a la comunidad afectiva y a la inversión emocional (Melucci, 1996: 72), a fin de promover la participación en la huelga entre las seguidoras de las cuentas.

Planteamos, a su vez, como hipótesis que estos colectivos utilizaron contenido propio para sus publicaciones, pero en el caso de Feminismos Madrid, por ser más minoritario y contar con menos recursos, acudió a los medios de comunicación y a otros colectivos para nutrirse de información que compartir con sus simpatizantes.

Con ello queremos explorar si en la convocatoria del $8 \mathrm{M}$ estos colectivos funcionaron como "organizaciones híbridas", que movilizan a sus miembros por medio de adhesiones de individuos que están conectados digitalmente y que favorecen la adaptación y la integración entre repertorios de acción colectiva más antiguos y más modernos, tanto online como offline (Chadwick y Dennis, 2017: 45). Veremos si la conexión digital fue capaz de favorecer la "proximidad virtual" (Gerbaudo, 2012: 32) entre quienes simpatizaban con la huelga. Indagaremos, a su vez, si estas cuentas contribuyeron a construir identidades colectivas comunes entre las participantes, de tal forma que la información que circuló por redes logró promover la politización de las reivindicaciones y la resistencia frente a las violencias que se ejercen hacia las mujeres. 


\section{Implicaciones de la dimensión digital en la acción colectiva}

La tensión entre las tendencias emancipatorias y represivas inherentes a la arquitectura de red de la comunicación digital, más allá del estéril debate entre las posibilidades de democratización o la reconfiguración de las estructuras de poder, es la que reconocen la mayoría de los estudiosos en este momento (Castells, 2009 y 2015; Dencik \& Leistert, 2015; Jordan, 2015, en Flesher y Gillan, 2017: 384 y 389). Atender a las posibilidades de emancipación implica contemplar cómo en el contexto de las democracias posindustriales más desarrolladas surgen formaciones de acción colectiva mediadas digitalmente más personalizadas, que crecen más rápido y son flexibles en el seguimiento de objetivos políticos en movimiento y en la solución de diferentes problemas (Bennett y Segerberg, 2012: 742).

Las conectividades, en este marco, se alimentan tanto de los encuentros cara a cara como de los mediados tecnológicamente. Los individuos se suman a las acciones masivas; pero la aproximación se produce más que a través de una identificación ideológica o grupal común, por medio de una expresión personal inclusiva y diversa (Bennett y Segerberg, 2012: 744). Las formaciones de acción conectivas a gran escala se apoyan en dos importantes elementos de "comunicación personalizada": el contenido político en forma de ideas sobre problemas comunes que son fácilmente personalizables y las tecnologías de comunicación personal, que permiten compartir y difundir esos temas mediante conexiones digitales con amistades o personas de confianza (Bennett y Segerberg, 2012: 744).

A partir de las posibilidades que ofrecen los medios digitales, en interacción con la personalización, el consumismo político y la política del "estilo de vida" posmaterialista, ha surgido el "movimiento de movilización híbrida” (Chadwick y Dennis, 2017: 45). El funcionamiento de las “organizaciones híbridas" es posible por la reconfiguración espacial y temporal de la vida política, a partir de la integración en su dimensión organizativa de la comunicación digital.

Esta comunicación favorece la adaptación y la integración entre repertorios de acción colectiva más antiguos y más modernos, tanto dentro como fuera de línea. Las organizaciones híbridas experimentan constantes cambios de forma, pues carecen de estructuras burocráticas y movilizan a sus miembros a través de adhesiones de individuos que están conectados digitalmente (Chadwick y Dennis, 2017: 45).

La fluidez de información que promueven los medios sociales elimina barreras para la acción colectiva, que se organiza en "estructuras postburocráticas” (Bimber, 2017: 13). En ellas, las sugerencias de participación pueden 
ser a partir de un comportamiento a solicitud de una organización movilizadora; por medio de una conducta socialmente inducida, en la cual las personas actúan porque otras lo hacen; o a través de un comportamiento autodirigido, motivado por la exposición a información política que no contenga solicitudes ni señales sociales de la conducta de otros ciudadanos (Bimber, 2017: 14-17).

La conexión digital sustituye prácticas interactivas en las "geografías públicas", permitiendo la "proximidad virtual”. Además de establecer lazos débiles frente a identidades colectivas fuertes, que en este contexto serían la excepción, dichas interacciones implican el riesgo de encerrarnos en una “cápsula” mediada (Gerbaudo, 2012: 32-33).

Siguiendo a Laclau (2005), Gerbaudo (2012) sostiene que la "proliferación de puntos de ruptura" que caracteriza a la experiencia social contemporánea, y que se resume en las formas de interacción subyacentes a los medios sociales, "hace formas políticas necesarias de agregación social" (Laclau, 2005: 230). "En ausencia de identidades incrustadas y redes sociales gruesas para sostener la acción colectiva, estos elementos deben crearse de manera proactiva y ad-hoc en el curso del proceso de movilización”. Para que la información práctica que circula por redes sociales en las reuniones colectivas virtuales no caiga en el vacío, es preciso que se construyan identidades colectivas comunes entre los participantes (Gerbaudo, 2012: 41).

De acuerdo con Melucci (1996: 72), esas identidades comunes implican una "inversión emocional" por parte de los participantes, más allá de la mera circulación de la información. Jasper (2011, en Gerbaudo, 2012: 41) destaca la importancia de una "energía emocional" como un recurso fundamental para las movilizaciones y la participación en protestas. Las redes sociales se han convertido en "conductos emocionales" para reconstruir el sentimiento de unidad entre individuos separados físicamente y para potenciar una narración emocional, capaz de motivar a la acción (Gerbaudo, 2012: 160-162).

\section{Metodología}

Como se señaló, en este artículo se pretende identificar el uso de la plataforma Facebook por parte de los movimientos sociales en periodos de alta intensidad informativa y de acción, como el 8 de marzo de 2018, de una manera cuantitativa. Asimismo, tenemos la intención de comprobar de qué forma los feminismos establecieron diálogos y alianzas para politizar reivindicaciones concretas respecto a las violencias machistas, los cuidados, la brecha salarial o el consumo. 
Para ello, se han analizado dos cuentas: Huelga Feminista $8 \mathrm{M}$ y Feminismos Madrid. El primer perfil, creado por la coordinadora estatal de la huelga feminista " 8 de marzo de 2018 " un año antes de esta fecha, se centró en difundir la información en torno a dicha convocatoria. Por otra parte, Feminismos Madrid, gestionado por la Comisión 8M Madrid, se define como "un espacio en la red para difundir las acciones conjuntas del movimiento feminista de Madrid". La relevancia de ambas cuentas reside en su centralidad para difundir la información sobre acciones y convocatorias alrededor de la huelga desde dos visiones diferentes: una central, que aglutina el trabajo de todas las comisiones a nivel estatal; y otra territorial, encargada de la organización y difusión de actividades en Madrid.

La muestra se seleccionó durante las dos semanas previas a la Huelga Feminista, entre el 22 de febrero y el 8 de marzo de 2018. El paro de mujeres, convocado internacionalmente, estaba motivado por la violencia machista, la desigualdad de género y las distintas formas de opresión contra las mujeres y tuvo un especial impacto en España, con más de 120 marchas en diferentes ciudades del país (Gómez, 2018), conformando un hito de movilización, apoyo y seguimiento masivo en el movimiento feminista.

Las unidades de análisis elegidas en este periodo fueron todas las publicaciones en ambos perfiles, independientemente de si estaban compuestas por textos, fotos o enlaces a otros eventos en Facebook o a noticias de medios de comunicación. En total se seleccionaron 388 publicaciones en las dos cuentas con un ritmo de difusión más elevado a medida que se acercaba el día 8 de marzo y una mayor presencia en el perfil de Huelga Feminista $8 \mathrm{M}$ (véase Tabla 1). ${ }^{3}$

El diseño metodológico incluyó tres variables centradas en el tipo de encuadre empleado en la publicación, su contribución al conocimiento sobre la convocatoria de paro o sobre el movimiento feminista y el origen de la información publicada. ${ }^{4}$ Las dos primeras variables fueron extraídas del libro de códigos propuesto por Ballesteros et al. (2017: 141) para el análisis de la interacción entre candidatos, partidos y ciudadanos en Facebook, durante la campaña de las elecciones generales en España en 2015. Sin embargo, dicho manual de codificación fue modificado, debido a las diferencias entre las características de los actores políticos estudiados (partidos y movimientos sociales) y al periodo de análisis (campaña electoral frente a un periodo previo a una convocatoria de huelga).

3 Todas las tablas se encuentran en el Anexo, al final del presente artículo (Nota del editor).

4 La primera versión del libro de códigos empleada por los codificadores contenía más variables como el uso de hashtags o el carácter de los recursos audiovisuales utilizados que fueron suprimidas del análisis final por diversas razones metodológicas. 
El estudio de los encuadres identificó si estos eran:

- Emocionales: si realizan apelaciones sentimentales y emotivas.

- Noticiosos: si asumen y muestran los marcos de los medios de comunicación.

- Logístico-movilizadores: si aportan informaciones sobre acciones y convocatorias en torno a la huelga.

- Didácticos: si explican las razones y los motivos del paro. ${ }^{5}$

La segunda de las variables se centró en explicar la contribución al conocimiento sobre la convocatoria de paro o sobre el movimiento feminista que realizan las publicaciones examinadas, distinguiendo entre: las que proporcionan información básica (sin ofrecer datos o justificaciones sobre la huelga), o argumentada (justificando la información con citas, datos, referencias y descripciones) (Varela, 2014; Giglietto y Selva, 2014, en Ballesteros et al., 2017: 141).

Por último, se incluyó una tercera variable enfocada en el origen de la información publicada, para detectar si las entradas eran de contenido propio, si pertenecían a medios de comunicación o provenían de activistas y organizaciones feministas. La inclusión de esta variable se debe a la necesidad de conocer: las redes de contacto y difusión en torno al movimiento, la identificación de las organizaciones como generadoras de contenido y la dependencia informativa de los medios convencionales.

Una vez creada la matriz de datos se realizó el tratamiento con el programa SPSS (V.22). Antes de efectuar los diferentes procedimientos y para medir la fiabilidad de los intercodificadores, se calculó el coeficiente de Kappa en el 15\% de la muestra, seleccionando aleatoriamente 58 unidades de análisis que fueron repartidas entre los dos codificadores que participaron en la investigación.

El único criterio de reparto fue que las unidades seleccionadas no hubieran sido codificadas por cada uno de ellos con anterioridad. El promedio entre las tres variables que componen la investigación fue de aproximadamente 0,8 , lo cual indica que el acuerdo entre codificadores fue elevado. Tras esta medida, se elaboraron tablas de contingencia que fueron sometidas a la prueba Chi Cuadrado, con la intención de determinar si existía una asociación estadísticamente significativa entre variables, así como al coeficiente Alfa de Cronbach, para medir la consistencia interna de dicha asociación.

5 En la primera versión del libro de códigos se proponía un encuadre adicional, juego estratégico, centrado en cómo los partidos y los candidatos se mostraban como ganadores o perdedores de las elecciones. Debido al carácter de nuestro caso de estudio, se consideró suprimir esta categoría (Ballesteros et al., 2017: 158). 


\section{Análisis}

\section{Encuadres dominantes}

La primera variable a examinar fue la de los marcos o encuadres en los cuales se inscribieron los post que se realizaron en las dos cuentas analizadas, en el periodo seleccionado (véase Tabla 2).

El enfoque más empleado en ambas cuentas fue el logístico y movilizador (en un $61,6 \%$ de los post publicados) y de una manera más intensiva en el caso del perfil de Huelga Feminista 8M (64,8\% frente a $50 \%$ en la cuenta de Feminismos Madrid). En los dos casos, pero sobre todo en el de Huelga Feminista 8M, las entradas se utilizaron para anunciar convocatorias de concentraciones, manifestaciones, asambleas, performances, talleres, charlas, mesas informativas, bicicletadas, caceroladas, acciones en mercados, universidades, centros sociales, etc. En este marco, un importante número de publicaciones ofrecieron instrucciones sobre cómo intervenir o colaborar en la huelga y aportaron información concreta sobre las convocatorias de diverso tipo que se realizaron antes y durante el paro. Si bien Huelga Feminista $8 \mathrm{M}$ hizo públicas las indicaciones de acciones distribuidas por toda la geografía española, Feminismos Madrid concentró las publicaciones en especial en el ámbito de la Comunidad de Madrid, dado el carácter regional de esta cuenta.

El frame emocional, en el 22\% de los casos, fue el segundo enfoque más usado. De una manera bastante similar en cuanto al porcentaje de entradas en las dos cuentas, aunque bastante por debajo del primer encuadre, las publicaciones trataron de generar solidaridad o identificación moral mediante apelaciones emotivas a las y los seguidores. Muestra de ello es, por ejemplo, la publicación de Huelga Feminista $8 \mathrm{M}$ de los versos reivindicativos de la poeta mexicana Jimena González, precedidos de la entrada "Porque nos escribimos y leemos mientras caminamos \#HaciaLaHuelgaFeminista” (Huelga Feminista 8M, 2018).

La creación del evento de Facebook "Huelga Feminista 8 de marzo" por parte de Feminismos Madrid se acompañó de un texto que invocaba emocionalmente a la participación a través de argumentarios como el siguiente:

Formamos parte de las luchas contra las violencias machistas, por el derecho a decidir sobre nuestro cuerpo y nuestra vida, para que se reconozca el valor, la dignidad y los derechos del trabajo doméstico y de cuidados, por la justicia social, la vivienda, la salud, la educación y un planeta sostenible. Porque somos pacifistas y estamos contra las guerras y las fronteras que desprecian la vida de miles de mujeres, niñas y niños y 
hombres. Y queremos unirnos para organizar la vida, la economía y las relaciones de otra forma (Feminismos Madrid, 2018).

Consideramos también que los numerosos videos y las cientos de fotografías de manifestaciones y concentraciones que se difundieron en las dos cuentas el 8 de marzo de 2018 contribuyeron a promover la participación y la adhesión a la convocatoria de huelga, recurriendo al componente emocional que implica representar a personas unidas compartiendo acciones e intervenciones diversas en pro de la misma reivindicación.

Encontramos que el tercer encuadre predominante fue el didáctico $(17,9 \%)$, el cual presentaba diferencias porcentuales importantes entre los dos casos de estudio, al ser empleado en mayor medida por el perfil de Feminismos Madrid (17,9\%) que por el de Huelga Feminista 8M (8,9\%). Las publicaciones que se inscribieron en este marco trataron de ofrecer explicaciones a la ciudadanía, mediante razonamientos o documentos, sobre cómo o por qué el movimiento feminista decidió adoptar una determinada posición o defender una medida.

Huelga Feminista 8M ofreció en algunas ocasiones explicaciones sobre cuestiones prácticas respecto a la huelga (sueldo que no se percibe, aspectos jurídicos, cómo hacer huelga de consumo, entre otras) y sobre los motivos para hacer el paro o acerca de la posición de los hombres en la convocatoria. En Feminismos Madrid se compartió periódicamente información sobre las claves de la huelga feminista, los porqués de la huelga laboral, la de consumo o la de cuidados, la cobertura legal, etcétera.

De igual modo, si bien el enfoque noticioso $(5,4 \%)$ era al que menos recurrieron los dos perfiles, también estuvo porcentualmente más presente en Feminismos Madrid (10,7\% frente a un 3,9\%). En este contexto, las publicaciones que se tuvieron en cuenta fueron las que contribuyeron a comprender, apoyar o rechazar hechos, propuestas, declaraciones, medidas o asuntos noticiosos que pudieran tener implicaciones políticas. Solo en contadas ocasiones, Huelga Feminista $8 \mathrm{M}$ compartió noticias publicadas en otros medios, las cuales, además, fueron sobre hechos controvertidos, como el despido de dos trabajadoras que habían anunciado que harían huelga (público.es, 2018) o las críticas a la actitud de los sindicatos mayoritarios CC.OO. y UGT ante la huelga en Extremadura (extremadura7dias. com, 2018).

La cuenta de Feminismos Madrid en Facebook hizo circular en este marco informaciones con calado político, como los datos de la Fiscalía en torno a la inexistencia de denuncias falsas sobre violencia en 2017, que desmontaban la posverdad de que las mujeres acusan falsamente a sus parejas o exparejas de ser violentadas (eldiario.es, 2017). 


\section{Contribución al conocimiento}

Para el análisis de la variable "Contribución al conocimiento" se valoraron, por un lado, las que consideramos "informaciones básicas"; esto es, aquellas que presentaban datos, hechos sobre la huelga y sus consecuencias apoyadas de citas o declaraciones, pero sin ofrecer razonamientos o explicaciones de apoyo. Por "informaciones argumentadas" entendimos los mensajes que aportaban información y opinión sobre la base de una argumentación sólida sostenida en citas o descripciones de la temática o el contenido.

$\mathrm{Al}$ igual que ocurre en los casos precedentes, si bien hay una asociación estadísticamente significativa entre el origen del post y el tipo de contribución al conocimiento, cabe destacar que se trata de una asociación débil (véase Tabla 3).

El 86,6\% de las entradas publicadas en los dos perfiles analizados contribuyeron de una manera básica al conocimiento. Este porcentaje fue menor en los post publicados en el perfil de Feminismos Madrid (el 75\% frente al 89,8\% del de Huelga Feminista 8M). Por el contrario, el porcentaje total de entradas que contribuyeron de manera argumentada $(13,4 \%)$ fue bajo en ambas cuentas, aunque estuvo más presente en el perfil de Feminismos Madrid (25\% frente a $10,2 \%)$.

Estas praxis se pueden explicar atendiendo a los encuadres en los que de forma más intensiva se insertan las entradas de las dos cuentas analizadas. Como se indicó antes, Feminismos Madrid tiene más publicaciones (porcentualmente hablando) que se pueden ubicar en los frames didáctico y noticioso que Huelga Feminista 8M. Esto supone que, además de tratar de activar contribuciones a la huelga ofreciendo información básica -que responde a un encuadre más logístico-movilizador-, Feminismos Madrid aporta informaciones que podrían perseguir fines más didácticos o divulgadores de cuestiones controvertidas o con implicación política para el movimiento feminista.

Por ejemplo, una entrevista sobre la legalidad de la huelga de 24 horas de cadenaser.com (2018) o un video de eldiario.es (2018) con las claves de la huelga, publicados el 23 de febrero de 2018 en la cuenta de Feminismos Madrid, estarían aportando información argumentada sobre por qué acudir a la huelga y de qué forma afrontarla en un contexto legal. También Huelga Feminista $8 \mathrm{M}$ contempla este tipo de publicaciones, aunque porcentualmente en menor medida, que suelen inscribirse en los frames didáctico, emocional o noticioso. Los diversos manifiestos que se hicieron públicos en esta cuenta en marzo de 2018, como el de las mujeres académicas, 
las periodistas valencianas o el colectivo de sanitarias, ofrecieron argumentaciones sobre por qué acudir a la huelga.

\section{Origen de las publicaciones}

Aquí la variable a estudiar era el origen del contenido publicado en las cuentas Huelga Feminista 8M y Feminismos Madrid en el periodo de 15 días previos al 8 de marzo de 2018 y durante esa fecha. Las posibles opciones a contemplar eran que la información fuera de creación propia, proveniente de medios de comunicación o de activistas y organizaciones de feministas (véase Tabla 4).

El origen del contenido publicado en la totalidad de las entradas de los dos perfiles estaba equilibrado entre el que pertenecía a activistas y organizaciones feministas $(46,9 \%)$ y el de creación propia (46,1\%). Sin embargo, en el perfil de Huelga Feminista $8 \mathrm{M}$ el contenido de creación propia fue superior $(51,6 \%)$ que en el caso de Feminismos Madrid (26,2\%). Esta tendencia es explicable, dado que el primer perfil direccionaba la información sobre convocatorias y acciones relacionadas con la huelga feminista a una web específicamente creada para tal evento y que suponía un nodo central de comunicación y un repositorio de información sobre actividades (hacialahuelgafeminista.org).

Por último, también cabe destacar cómo el perfil de Feminismos Madrid empleó de manera más intensiva (17,9\% frente al 3,9\%) el contenido que provenía de medios de comunicación sensibles con las causas feministas como eldiario.es, la Cadena Ser, el diario El Salto, publico.es o Pikara Magazine.

\section{Conclusiones}

En relación con los resultados obtenidos, se constató una asociación estadísticamente significativa. Esto implica que la publicación en las cuentas analizadas se relaciona con un enfoque determinado, un tipo de contribución al conocimiento y un origen concreto del contenido que se publica. Así, podemos concluir que el perfil en Facebook de Huelga Feminista 8M argumenta de una forma más básica, prima el contenido propio y emplea sobre todo el frame logístico-movilizador unido al emocional. Por su parte, Feminismos Madrid contribuye al conocimiento de una manera más argumentada, prima el contenido de activistas y organizaciones feministas y también utiliza el encuadre logístico-movilizador, aunque en un porcentaje muy elevado también el didáctico. 
El análisis nos permite inferir que el componente afectivo y la energía emocional no fueron vehículos fundamentales para promover las movilizaciones. Las "organizaciones híbridas" que constituyeron las dos cuentas analizadas contribuyeron sobre todo a aproximar virtualmente a las personas con inquietudes en la huelga, a partir de la mediación digital, anunciando de forma masiva la diversidad de convocatorias que se produjeron en el ámbito digital, pero en especial fuera de línea. Este hecho ratifica lo expuesto por Bennet y Segerberg (2012: 744) al indicar cómo estas conectividades se alimentan dentro y fuera de la red con interacciones cara a cara, a menudo intensas y que tienen lugar en diferentes espacios (plazas, campamentos, asambleas).

La fluidez de la información que promovieron Huelga Feminista 8M y Feminismos Madrid trató de eliminar barreras para la acción colectiva que, como indica Bimber (2017:13), se organiza en "estructuras postburocráticas". El hecho de que el frame dominante fuera el logístico-movilizador pudo impulsar que la sugerencia de participación en la convocatoria de huelga se promoviera a solicitud de una organización movilizadora, como las dos cuentas estudiadas.

Se concluye, a la vista del masivo respaldo de la primera huelga feminista en España y del apoyo y seguimiento a estas cuentas, que la información práctica que se difundió a través de Facebook no cayó en el vacío. Informar de manera profusa sobre las convocatorias, apelar emocionalmente a la participación, aportar información práctica sobre las causas y las consecuencias de la huelga y realizar un aporte noticioso de creación propia y ajena supuso un desafío a la "interpasividad" o a la evitación de una acción real para ejercer la resistencia activa. El tejido de alianzas y diálogos en constante ebullición antes y durante la huelga fue capaz de contribuir a seguir creando una comunidad feminista, dispuesta a politizar los motivos de la convocatoria y a ejercer resistencia dentro y fuera de la Red.

\section{Referencias}

Ballesteros, Carlos et al. (2017), "La interacción entre candidatos, partidos y ciudadanos en Facebook durante la campaña de las Elecciones Generales de 2015. Un análisis Cuantiativo", en Dader, José Luis y Campos, Eva [coords.], La búsqueda digital del voto. Cibercampañas Electorales en España 2015-2016, España: Tirant.

Banet-Weiser, Sarah y Miltner, Kate (2016), “\#MasculinitySoFragile: Culture, Structure, and Networked Misogyny”, en Feminist Media Studies, vol. 16, núm. 1. Disponible en: https://www.tandfonline.com/doi/abs/10.1080/14680777.2016.1120490?journalC ode $=$ rfms20 [13 de marzo de 2018]. 
Bennett, Lance y Segerberg, Alexandra (2012), “The logic of connective action. Digital media and the personalization of contentious politics", en Information, Communication \& Society, vol. 15, núm. 5. Disponible en: https://www.tandfonline.com/doi/abs/10.1 080/1369118X.2012.670661 [15 de febrero de 2018].

Bimber, Bruce (2017), "Three Prompts for Collective Action in the Context of Digital Media”, en Political Communication, vol. 34, núm. 1. Disponible en: https://www. tandfonline.com/doi/abs/10.1080/10584609.2016.1223772? journalCode $=$ upcp20 [15 de febrero de 2018].

Braidotti, Rosi (2004), "El ciberfeminismo con una diferencia”, en Fischer Pfeiffer, Amalia [ed.], Feminismo, diferencia sexual y subjetividad nómade, España: Gedisa.

cadenaser.com (2018), "Si los hombres paran el 8 de marzo sería una huelga general, se pierde el objetivo feminista”, en cadenaser.com. Disponible en: http://cadenaser.com/ programa/2018/02/23/hoy_por_hoy/1519372392_073096.amp.html [13 de marzo de 2018].

Castells, Manuel (2009), Communication Power, Reino Unido: Oxford University Press.

Castells, M. (2015), Networks of Outrage and Hope: Social Movements in the Internet Age, Reino Unido: Polity Press.

Chadwick, Andrew y Dennis, James (2017), "Social Media, Professional Media and Mobilisation in Contemporary Britain: Explaining the Strengths and Weaknesses of the Citizens Movement 38 Degrees", en Political Studies, vol. 65, núm.1. Disponible en: http://journals.sagepub.com/doi/abs/10.1177/0032321716631350 [20 de enero de 2018].

CIS [Centro de Investigaciones Sociológicas] (2018), Barómetro de mayo de 2018, Estudio num. 3213, España: Centro de Investigaciones Sociológicas. Disponible en: http:// www.cis.es/cis/export/sites/default/-Archivos/Marginales/3200_3219/3213/ es3213mar.pdf [25 de mayo de 2018].

Dean, Jodi (2005), "Communicative Capitalism: Circulation and the Foreclosure of Politics", en Cultural Politics, vol. 1, núm. 1. Disponible en: https://read.dukeupress. edu/cultural-politics/article-abstract/1/1/51/34262/Communicative-CapitalismCirculation-and-the [20 de enero de 2018].

Dean, Jonathan y Aune, Kristin (2015), "Feminism Resurgent? Mapping Contemporary Feminist Activisms in Europe”, en Social Movement Studies, vol. 14, núm. 4. Disponible en: https://www.tandfonline.com/doi/abs/10.1080/14742837.2015.1077112 [20 de enero de 2018].

Dencik, Lina y Leistert, Oliver [eds.] (2015), Critical perspectives on social media and protest: Between control and emancipation, Inglaterra: Rowman \& Littlefield.

eldiario.es (2017), "La Fiscalía no probó ninguna denuncia falsa por violencia de género el año pasado", en eldiario.es. Disponible en https://www.eldiario.es/sociedad/violencia_ de_genero-machismo-asesinatos_machistas-denuncias_falsas_0_683832174.html [13 de mayo de 2018].

eldiario.es (2018), "El 8M, todas a la huelga" [video online]. Disponible en: https://www. youtube.com/watch?v=AMKbQZIxOVs\&feature=youtu.be [13 de mayo de 2018].

extremadura7dias.com (2018), "La Administración, CCOO y UGT tratan de boicotear la huelga feminista", en extremadura7dias.com. Disponible en: http://www. extremadura7dias.com/noticia/la-administracion-ccoo-y-ugt-tratan-de-boicotear-lahuelga-feminista [13 de mayo de 2018]. 
Diana Fernández-Romero y José Manuel Sánchez-Duarte. Alianzas y resistencias feministas en Facebook para la convocatoria del 8M en España

Evans, Elizabeth y Chamberlain, Prudence (2015), "Critical Waves: Exploring Feminist Identity, Discourse and Praxis in Western Feminism”, en Social Movement Studies, vol. 14, núm. 4. Disponible en: https://www.tandfonline.com/doi/abs/10.1080/1474283 7.2014.964199? journalCode $=$ csms20 [15 de enero de 2018].

Favavo, Laura (2018), “Los Estudios Críticos de Internet: conceptos, debates y retos”, en Teknokultura, vol. 15, núm. 1. DOI: 10.5209/TEKN.56687 Disponible en: https://doi.org/10.5209/TEKN.56687 [3 de junio de 2018].

Favaro, Laura (2017), “Transnational Technologies of Gender and Mediated Intimacy”, [tesis doctoral], Inglaterra: University of London.

Feminismos Madrid (2018), "Huelga feminista 8 de marzo". Disponible en: https://www. facebook.com/events/284650828735354/ [25 de abril de 2018].

Fernández-Romero, Diana y Núñez-Puente, Sonia (2017), "Producciones activistas online de la víctima de violencia como sujeto político. El paro internacional de mujeres \#8M", en Sierra Sánchez, Javier [coord.], Nuevas tecnologias audiovisuales para nuevas narrativas interactivas digitales en la era multidispositivo, España: McGraw-Hill Interamericana de España.

Flesher, Cristina y Gillan, Kevin (2017), "Navigating the technology-media-movements complex", en Social Movement Studies, vol. 16, núm. 4. Disponible en: https://www. tandfonline.com/doi/abs/10.1080/14742837.2017.1338943? journalCode $=$ csms20 [4 de abril de 2018].

Gerbaudo, Paolo (2012), Tweets and the Streets: Social Media and Contemporary Activism, Inglaterra: Pluto Press.

Gómez, Manuel (2018), "Movilización histórica por la igualdad de las mujeres", en El Pais. Disponible en: https://elpais.com/economia/2018/03/08/ actualidad/1520545956_654616.html [25 de marzo de 2018].

Huelga Feminista 8M (2018), "Desde México nos llegan los versos de una joven poeta, Jimena González”. Disponible en: https://es-la.facebook.com/HuelgaFeminista/ posts/desde-m\%C3\%A9xico-nos-llegan-los-versos-de-una-joven-poeta-jimenagonz\%C3\%A1lezporque-nos-/196958867724178/ [25 de mayo de 2018].

IAB Spain (2018), “Estudio Anual de Redes Sociales”. Disponible en: https://iabspain. es/wp-content/uploads/estudio-redes-sociales-2018_vreducida.pdf [ 25 de junio de 2018].

Jordan, Tim (2015), Information politics: liberation and exploitation in the digital society, Inglaterra: Pluto Press.

Keller, Jessalynn (2015), Girls Feminist Blogging in a Postfeminist Age, Estados Unidos: Routledge.

Laclau, Ernesto (2005), La razón populista, España: Fondo de Cultura Económica.

McRobbie, Angela (2017), "Post-feminismo y cultura popular: Bridget Jones y el nuevo régimen de género”, en Investigaciones Feministas, vol. 8, núm. 2. DOI: 10.5209/INFE.58316 Disponible en: https://doi.org/10.5209/INFE.58316 [20 de junio de 2018].

Melucci, Alberto (1996), Challenging Codes: Collective Action in the Information Age, Reino Unido: Cambridge University Press.

Núñez-Puente, Sonia y Fernández-Romero, Diana (2017), “Narrativas transformadoras y testimonio ético: las estrategias discursivas de la Plataforma Feminista 7N, Contra las Violencias Machistas", en index.comunicación, vol. 7, núm. 3. Disponible en: 
http://journals.sfu.ca/indexcomunicacion/index.php/indexcomunicacion/article/ viewFile/341/330 [15 de febrero de 2018].

Núñez-Puente, Sonia y Fernández-Romero, Diana (2017), "Desafíos de las tecnologías del activismo feminista: reapropiaciones del cuerpo femenino en Youtube", en Labrys, estudos feministas/études feministas, núm. 30. Disponible en: https://www.labrys.net. br/labrys30/sumarios/patriarcat.htm [ 15 de febrero de 2018].

Núñez-Puente, Sonia y Gámez-Fuentes, María José (2017), "Spanish feminism, popular misogyny and the place of the victim", en Feminist Media Studies, vol. 17, núm. 5. Disponible en: https://www.tandfonline.com/doi/abs/10.1080/14680777.2017.135 0527 [25 de noviembre de 2017].

Perdomo, Inmaculada (2016), "Género y tecnologías. Ciberfeminismos y construcción de la tecnocultura actual", en Revista Iberoamericana de Ciencia, Tecnologia y Sociedad, vol. 31, núm. 11. Disponible en: http://www.revistacts.net/files/Volumen_10_ Numero_31/PerdomoEDITADO.pdf [25 de abril de 2018].

Pérez, Lourdes y Revuelta-Domínguez, Francisco Ignacio (2008), "Ciberfeminism/o. La manifestación tecnológica de las mujeres", en Maya Frades, Valentina [ed.], Mujeres Rurales. Estudios multidisciplinares de género, España: Universidad de Salamanca.

publico.es (2018), "Dos trabajadoras denuncian haber sido despedidas por una subcontrata de Páginas Amarillas tras informar de que harán huelga", en publico.es. Disponible en: http://www.publico.es/economia/trabajadoras-denuncian-haber-sido-despedidassubcontrata-paginas-amarillas-informar-haran-huelga.html [13 de mayo de 2018].

Reverter, Sonia (2013), "Ciberfeminismo: de virtual a político", en Teknokultura, vol. 10, núm. 2. Disponible en: http://repositori.uji.es/xmlui/handle/10234/84352 [13 de marzo de 2018].

Sánchez-Duarte, José Manuel (2016), "The Net as a space for political militancy: technology and participation in the electoral campaign", en Communication \& society, vol. 29, núm. 3. DOI: 10.15581/003.29.3.sp.33-47 Disponible en: http://dx.doi. org/10.15581/003.29.3.sp.33-47 [13 de marzo de 2018].

Sánchez-Duarte, José Manuel y Fernández-Romero, Diana (2017), "Subactivismo feminista y repertorios de acción colectiva digitales: prácticas ciberfeministas en Twitter", en El profesional de la información, vol. 26, núm. 5. Disponible en: http://www. elprofesionaldelainformacion.com/contenidos/2017/sep/11.pdf [13 de marzo de 2018].

Valera, Lidia (2014), "El debate público en la blogosfera política española durante la campaña electoral de 2011: ¿Hacia un debate público enriquecido o fragmentado?”, en Tripodos, núm. 34. Disponible en: http://www.tripodos.com/index.php/Facultat_ Comunicacio_Blanquerna/article/view/170 [13 de marzo de 2018].

Vincos.it (2018), "World Map of Social Networks". Disponible en: http://vincos.it/worldmap-of-social-networks/ [ 25 de junio de 2018].

Wilding, Faith (2004), “¿Dónde está el feminismo en el ciberfeminismo?”, en Lectora, núm. 10. Disponible en: http://revistes.ub.edu/index.php/lectora/article/view/7069 [15 de marzo de 2018].

Zafra, Remedios (2018), “Redes y (Ciber) Feminismos. La revolución de la representación que derivó en alianza", en Revista Dígitos, núm. 4. Disponible en: https://revistadigitos. com/index.php/digitos/article/view/116 [20 de junio de 2018].

Zizek, Slavoj (1997), The Plague of Fantasies, Inglaterra: Verso. 
Anexo

Tabla 1

Publicaciones analizadas y distribución por perfiles

Huelga Feminista 8M

Feminismos Madrid

Número de post
Fuente: Elaboración propia.

304

84

Tabla 2

Tipo de frames empleados según el origen del post

\begin{tabular}{lcc}
\hline & Huelga Feminista 8M & Feminismos Madrid \\
\hline Emocional & $22,4 \%$ & $21,4 \%$ \\
\hline Noticioso & $3,9 \%$ & $10,7 \%$ \\
\hline Logístico-movilizador & $64,8 \%$ & $50 \%$ \\
\hline Didáctico & $8,9 \%$ & $17,9 \%$ \\
\hline & $\mathrm{N}=304$ & $\mathrm{~N}=84$ \\
\hline
\end{tabular}

$\operatorname{Chi}^{2}(\mathrm{~N}=388)=12,834, \mathrm{p}=.005$

$\mathrm{V}$ de Cramer $=, 182, \mathrm{p}=.005$

Fuente: Elaboración propia. 


\section{Tabla 3}

Tipo de contribución al conocimiento según el origen del post

\begin{tabular}{lcc}
\hline & Huelga Feminista 8M & Feminismos Madrid \\
\hline Básica & $89,8 \%$ & $75 \%$ \\
\hline Argumentada & $10,2 \%$ & $25 \%$ \\
\hline & $\mathrm{N}=304$ & $\mathrm{~N}=84$ \\
\hline
\end{tabular}

$\mathrm{Chi}^{2}(\mathrm{~N}=388)=12,426, \mathrm{p}<.001$

$\mathrm{V}$ de Cramer=,179, $\mathrm{p}<.001$

Fuente: Elaboración propia.

Tabla 4

Origen del contenido publicado según el origen del post

\begin{tabular}{lcc}
\hline & Huelga Feminista 8M & Feminismos Madrid \\
\hline Contenido propio & $51,6 \%$ & 26,2 \\
\hline Medios de comunicación & $3,9 \%$ & $17,9 \%$ \\
\hline $\begin{array}{l}\text { Activistas y organizaciones } \\
\text { de feministas }\end{array}$ & $44,4 \%$ & $56 \%$ \\
\hline & $\mathrm{N}=304$ & $\mathrm{~N}=84$ \\
\hline
\end{tabular}

$\operatorname{Chi}^{2}(\mathrm{~N}=388)=29,412, \mathrm{p}<.001$

$\mathrm{V}$ de Cramer $=, 275, \mathrm{p}<.001$

Fuente: Elaboración propia. 
Diana Fernández-Romero. Doctora en Ciencias de la Información por la Universidad Complutense de Madrid. Especialista Universitaria en Cultura y Violencia de Género por la Universidad Nacional de Educación a Distancia. Docente en la Facultad de Ciencias de la Comunicación de la Universidad Rey Juan Carlos, Madrid, España. Líneas de investigación: comunicación, género, estudios sobre el discurso, ciberfeminismo. Publicaciones recientes: Peñamarín, Cristina y Fernández Romero, Diana (en prensa, 2019), "Women Survivors of Nullification by Sexist Abuse. The Role of Affects of Proximity and Recognition", en Gámez Fuentes, María José, Núñez Puente, Sonia and Gómez Nicolau, Emma [eds.], Re-writing Women as Victims: From Theory to Practice, Inglaterra: Routlegde; Fernández-Romero, Diana (2019). Spain's campaigns against gender violence: the effect on abused women's identities from the victim-agent dichotomy. Journal of Gender Studies, 0(0), 1-15; Núñez-Puente, Sonia, D’ Antonio, Sergio, Fernández-Romero, Diana (en prensa, 2019). Twitter Activism and Ethical Witnessing: Possibilities and Challenges of Feminist Politics Against Gender-Based Violence. Social Science Computer Review.

José Manuel Sánchez-Duarte. Doctor en Comunicación por la Universidad Rey Juan Carlos de Madrid y en Ciencias Sociais (especialidad Políticas) por la Pontifícia Universidade Católica de São Paulo. Docente en la Facultad de Ciencias Jurídicas y Sociales, Universidad Rey Juan Carlos, Madrid, España. Líneas de investigación: comunicación política, ciudadanía digital, participación política. Publicaciones recientes: Sánchez Duarte, José Manuel y Fernández Romero, Diana (2017), "Subactivismo feminista y repertorios de acciones colectiva digitales: prácticas ciberfeministas en Twitter”, en El profesional de la información, vol. 26, núm. 5. Disponible en: https://recyt. fecyt.es/index.php/EPI/article/viewFile/epi.2017.sep.11/36480; Sánchez Duarte, José Manuel (2016), “The Net as a space for political militancy: technology and participation in the electoral campaign", en Communication \& Society, vol. 29, núm. 3. Disponible en: https://www.unav.es/fcom/communication-society/en/articulo.php?art_id=579; Sánchez Duarte, José Manuel y Magallón Rosa, Raúl (2016), "Estrategias de organización y acción política digital", en Revista de la Asociación Española de Investigación de la Comunicación, vol. 3, núm. 5. Disponible en: http://www.revistaeic.eu/index.php/raeic/article/view/115. 